\title{
Das Problem der Willensfreiheit und die Psychoanalyse
}

\section{Das Es, das Ich und das Über-Ich}

Die Tiefenpsychologie Freuds hat unsere Anschauungen über psychische Vorgänge so grundlegend verändert, dass es unmöglich erscheint, an das Problem der Willensfreiheit heranzutreten, ohne die Ergebnisse der Forschungsarbeit Freuds zu berücksichtigen.

Das ganze seelische Leben des Menschen wird von Freud dem Lustprinzip untergeordnet und das Gefühl der Unlust als eine gewisse innere Erregung bzw. Spannung definiert, die nach Abfuhr drängt.

Wenn wir uns die menschlichen Handlungen näher ansehen, bemerken wir tatsächlich, dass dieses Prinzip unser ganzes Tun und Lassen bestimmt. Denn bei jeder menschlichen Handlung besteht die Betätigung des Willens im Abwägen der Lust- und Unlustgefühle, die mit dieser Handlung verbunden sind.

Das Lustprinzip erfährt nach Freud insoferne eine Einschränkung, als der Mensch auf seine Umwelt Rücksicht nehmen muss. Er kann also seinen Gefühlen nicht freien Lauf lassen, sondern muss sie mit den Anforderungen der Aussenwelt koordinieren. Der Repräsentant des Gefühlslebens ist das Unbewusste, welches Freud das Es nennt. Das Ich ist für Freud nicht der Inbegriff der Gesamtpersönlichkeit, weil er eine Dreiteilung vornimmt und zwischen Es, Ich und Über-Ich unterscheidet.

Die durch das Realitätsprinzip aufgezwungene Kontrolltätigkeit wird durch dieses Ich ausgeübt. Es ist auch die Instanz, durch die unliebsame, mit den Forderungen der Realität nicht zu vereinbarende Triebregungen und mit diesen zusammenhängende Vorstellungen verdrängt werden, was dann unter Umständen zu seelischen Erkrankungen führt, deren Wesen darin besteht, dass das Verdrängte immer wieder zur Oberfläche, zum Bewusstsein, vorstosst, vom Ich aber niedergehalten wird.

Das Ich ist also für Freud der Repräsentant der Realität und jene Instanz, die vor allem die Eindrücke der Aussenwelt in sich aufnimmt und sie verarbeitet. Freud nennt daher das Ich auch «Wahrnehmungssystem». Ob eine von mir zu setzende Handlung im Endergebnis für mich eine Lust oder aber Unlustquelle sein wird, hängt nicht nur von meiner Objektbesetzung, sondern 
auch davon ab, ob bestimmte in der Aussenwelt liegende Voraussetzungen eintreffen werden oder nicht. Man kann ja nicht in die Zukunft blicken und muss sich daher mit minderen oder grösseren Wahrscheinlichkeiten begnügen. Es ist die Aufgabe des Ich, sie richtig einzuschätzen. Dabei ist es erstaunlich, wie sich das Ich bei dieser Beurteilung der Wirklichkeit vom Es beeinflussen lässt.

Obwohl nun die Beurteilungen des Ich im starken Masse vom Gefühlsmässigen beeinflusst werden, daher auch seine Entscheidungen, ist es doch jene Instanz, die sich bemüht, objektiv zu sein und die Innenwelt des Menschen der Aussenwelt anzupassen. Das Ich übt eine kontrollierende Tätigkeit aus, es erwägt verschiedene Möglichkeiten, vergleicht sie und trifft schliesslich die Wahl, die es für richtig hält. Es empfängt nicht nur Eindrücke der Aussenwelt, es verarbeitet diese auch gedanklich und bedient sich dabei der Logik, deren Gesetze es für sich als ausnahmslos bindend anerkennt. Es kann unter richtiger Einsicht nicht nur Methoden ändern, die zum Ziele führen, sondern es kann auch Objektbesetzungen unter richtiger Einsicht aufgeben und nach anderen, passenderen, Ausschau halten. Der Repräsentant des Ethischen, der sittlichen Forderung, ist Freud zufolge das Über-Ich, unter dem er das Gewissen versteht. Das Ich kann nun auch dem Über-Ich gegenüber eine kritische Stellung einnehmen und unter besserer Einsicht seine Ideale revidieren und von den herkömmlichen Anschauungen vom «Soll» zu neueren, welche der objektiven Lage besser entsprechen, übergehen.

Was die Entstehung des Über-Ich's betrifft, meint Freud, dass sich im Laufe der Zeit ein Teil des Ich abgesondert und gewissermassen selbständig gemacht hat. Man weiss ja, welche Bedeutung die Psychoanalyse dem Einfluss des Vaters im Leben eines jeden einzelnen beimisst. In der Stimme des Gewissens vernehmen wir nicht nur die Stimme des Vaters, sondern der ganzen Reihe unserer Vorfahren, die uns gewisse Verbote auferlegten. Es handelt sich hier um ein Erbgut, welches tief im Unbewussten verankert ist. Man erinnere sich nur an den Roman Dostojewskys «Schuld und Sühne», in dem uns der Dichter zeigt, wie machtlos auch die noch so fein ausgeklügelten Argumente unseres bewussten Denkens gegenüber der Stimme des Gewissens sind.

So ist unser Ich einerseits den Vorstössen des Es, andererseits den Anforderungen des Über-Ich ständig ausgesetzt. Die daraus entstehenden Konflikte bilden die nie versiegende Quelle, aus der die Dichtung schöpft. Auf der gleichen Grundlage entstehen aber auch die zahllosen Abweichungen von der Norm auf psychischem Gebiet. Es handelt sich hierbei um Konflikte zwischen dem Ich und dem Es einerseits und dem Ich und Über-Ich, oder wie Freud es noch nennt, dem Ich-Ideal andererseits. Auf den Konflikten erster 
Art beruhen nach Freud die Neurosen, auf Konflikten der zweiten Art die Psychosen, deren Hauptmerkmal darin besteht, dass das Ich die Realität fallen lässt. Wir sehen also, dass unsere Gefühle und Affekte in tieferen, dem Bewusstsein weniger zugänglichen Bereichen verborgen sind, wogegen die rationalisierende Tätigkeit von unserem Bewusstsein, dessen Repräsentant das Ich ist, ausgeht.

Aber auch dann, wenn wir das Es für sich allein betrachten, erhalten wir kein einheitliches Bild. Der Kampf zwischen Liebe und Hass, zwischen den libidinösen und den agressiven Trieben, von denen später noch mehr zu sagen sein wird, geht auch hier stets vor sich.

Der Mensch ist eben kein homogenes Wesen; in seiner Psyche sind Kräfte vorhanden, die sich nach verschiedenen, oft entgegengesetzten Richtungen, betätigen. Das Zustandekommen einer Entscheidung ist demnach das Ergebnis des Zusammenwirkens aller dieser Kräfte, sozusagen ihre mittlere Linie. Das Ich bemüht sich unter Berücksichtigung der Realität, diese mittlere Linie zu finden und sind für uns nur diese Bemühungen des Ich und deren Ergebnis sichtbar. Die Kräfte, die dahinter verborgen sind und die eigentlich diese Entscheidungen herbeiführen, sehen wir nicht. Die vielen Faktoren, die eine Entscheidung herbeiführen, aber auch ihr gegenseitiges Verhältnis bleiben uns verborgen und unsichtbar, weshalb uns auch die getroffene Entscheidung ausschliesslich in einer «freien» Willensäusserung begründet erscheint. Sehr zutreffend heisst es in der Abhandlung «Der Verbrecher und seine Richter» von Franz Alexander und Hugo Staub: «Wir meinen, dass der Begriff des freien Willens nichts anderes bedeutet, als den narzistischen Wunsch, ja das Postulat der Moralisten, dass das Über-Ich den seelischen Apparat des Menschen uneingeschränkt befehligen möge. Sie meinen, der Mensch habe immer die freie Wahl zwischen Gut und Böse, und diese Vorstellung der freien Wahl sei die Grundlage der Verantwortung, wenn wir falsch handeln, so sind wir verantwortlich, weil wir ja auch anders hätten handeln können.

Allerdings, wenn im Moment des Handelns das Kräfteverhältnis der verschiedenen Motive ein anderes gewesen wäre. Aber dieses die Handlung bestimmende Kräfteverhältnis der verschiedenen Motive ist von dem Gesamterleben des einzelnen, von seiner persönlichen Lebensgeschichte und seiner Konstitution abhängig. Hätte er einen anderen Vater gehabt, hätte er eine andere Erziehung genossen, hätte er vor der Tat ein Glas Bier weniger getrunken, so wäre seine Handlung anders ausgefallen. Der freie Wille der Moralisten und Philosophen bedeutet nicht nur die einseitige Berücksichtigung nur der bewussten Motive, sondern noch mehr als das, die Annahme eines in dem Weltgeschehen frei schwebenden moralischen Machtfaktors, der das 
psychische Geschehen jederzeit beeinflussen kann, aber selbst eine von jedem Einfluss unabhängige Existenz führt». ${ }^{1}$

Trotz dieser Unübersichtlichkeit und Vielfältigkeit der Motive, können wir, zumindest theoretisch, zweierlei Vorgänge unterscheiden:

1. Die gefühlsmässigen, d.h. den Widerstreit der Lust- und Unlustgefühle, ganz gleich, ob es sich um die triebhaften Äusserungen des Es mit seiner Erotik oder aber um die ethischen Gebote des Über-Ichs handelt.

2. Die verstandesmässigen, d.h. die kritisierende und kontrollierende Tätigkeit des Ichs, als Repräsentant der Forderungen der Realität.

Wie schon ausgeführt wurde, ist es das Es, welches mit dem physischen Substrat eng verwachsen ist und, aus ihm hervorgehend, die starken Ichtriebe, oder wie man sie allgemein nennt, die Selbsterhaltungstriebe, aber auch die mit so viel Energie behafteten libidinösen, d.h. erotischen Triebregungen entwickelt. Das Es «besetzt» Objekte, d.h. bemächtigt sich ihrer, wobei diese Besetzung von einem Objekt auf ein anderes übertragen werden kann. Hierher gehört auch der «Narzismus», unter dem Freud die Liebe zum eigenen Körper versteht und den er als eine libidinöse Ergänzung des Selbsterhaltungstriebes ansieht. Ebenso die Agressionstriebe. Gilt doch ein Totschlag, also eine Agression, unter dem Druck eines «unwiderstehlichen Zwanges» begangen, auch nach dem geltenden Strafrecht als unter Ausschluss des «freien» Willens verübt.

Das Es ist nach Freud amoralisch, das Ich ist bemüht moralisch zu sein und das Über-Ich kann hypermoralisch und dann so grausam werden, wie nur das Es.

In der bereits erwähnten Abhandlung «Der Verbrecher und seine Richter» von Franz Alexander und Hugo Staub wird u.a. ausgeführt, dass der Mensch als «kriminelles» d.h. nicht sozial angepasstes Wesen auf die Welt kommt und in den ersten Lebensjahren seine Kriminalität in fast vollem Umfange beibehält. Seine eigentliche soziale Anpassung beginnt erst nach dem 4. bis 6. Lebensjahre. Die Autoren des angeführten Werkes meinen, dass man diese Tatsache sich am besten durch ein «Gedankenexperiment» vergegenwärtigen kann, das darin besteht, dass man sich eine Welt vorstellt, in der die Kinder zwischen dem 2. und 6. Lebensjahre in ihrer physischen Kraft den Erwachsenen so überlegen wären, wie heute die Erwachsenen den Kindern und die ersteren alle ihre Phantasien in Handlungen umsetzen könnten. Diese Riesenkinder Gullivers würden im Zwergenlande der Erwachsenen eine Welt der reinprozentigen Kriminalität verwirklichen. 
Wir sehen also, dass insoweit wir es mit dem Triebhaften im Menschen zu tun haben, uns die Beantwortung der Frage, ob der menschliche Wille frei oder unfrei sei, keine Schwierigkeiten bereitet. Von einem Menschen, den man als den «Sklaven» seiner Leidenschaften bezeichnet, wird niemand behaupten, dass er aus «freien Stücken» handelt.

Wie verhält es sich aber dort, wo das Über-Ich an unseren Handlungen beteiligt ist? Hier scheint die Sachlage nicht so durchsichtig zu sein, weshalb wir uns auch mit dem Über-Ich und den Faktoren, die bei seiner Errichtung massgebend waren, näher beschäftigen müssen.

\section{Das Über-Ich und der freie Wille}

Worin das Wesen des Über-Ichs im grossen und ganzen besteht, haben wir vorhin dargelegt. Das Über-Ich stellt gewisse Verbote auf, deren Übertretung unlustvolle Schuldgefühle auslöst. In seinem Werk «Das Ich und das Es» definiert Freud dieses Schuldgefühl als eine Spannung zwischen den Ansprüchen des Gewissens und den Leistungen des Ich. An einer anderen Stelle desselben Werkes drückt er sich noch genauer aus, indem er sagt, dass es sich hiebei um die Kritik des Über-Ich handelt, welche im Ich wahrgenommen wird.

Was das Lustprinzip betrifft, kann man sagen, dass für das Es die positive Seite, d.h. die Gewinnung von Lustgefühlen, bzw. die Vermeidung der Unlust das charakteristische ist; für das Über-Ich hingegen die negative Seite desselben Prinzips, d.h. die Zufügung eines Unlustgefühls als Strafe für die Übertretung eines Gebotes. Beim Es entsteht zuerst ein Zustand des Reizes, bzw. der Erregung, der als unangenehm empfunden wird und dessen Aufhebung durch die Triebbefriedigung von positiven Lustgefühlen begleitet ist. Beim Über-Ich ist der Hergang gewissermassen ein umgekehrter. Das Es setzt sich trotz der vom Über-Ich aufgestellten Verbote durch, was dann unlustbetonte Reuegefühle erzeugt. Das Ich versucht zwischen den beiden sozusagen zu vermitteln, möchte einen Kompromiss schliessen, doch nützen die nachträglichen Rationalisierungen und Beschwichtigungen des Ich nicht mehr viel.

Die Strafe muss eben abgebüsst werden, damit ein Gleichgewichtszustand wiederhergestellt wird. Es kann auch sein, dass die böse Tat gar nicht zur Ausführung gelangt, dass aber schon der Wunsch, sie zu begehen, genügt, um ein Schuldbewusstsein wachzurufen. Darauf werden wir noch später zurückkommen.

Das Über-Ich verhängt nicht nur Strafen in Form von Reue- und Schuldgefühlen, es betätigt sich auch positiv. Unter seinem Einfluss vollzieht sich 
die sogenannte «Sublimierung», die darin besteht, dass die Libido von niedrigeren auf höhere Ziele abgelenkt wird, wobei ursprünglich asoziale Gefühle allmählich in den Dienst der Gesellschaft gestellt werden. So erfährt z.B. nach Ansicht Freuds die sadistische Komponente bei dem Beruf des Chirurgen und des Staatsanwaltes eine solche Sublimierung, wobei aber auch der verdrängte und nicht sublimierte Rest dieser Komponente unbewusst auf seine Rechnung kommt.

In seiner Abhandlung «Das Unbehagen in der Kultur» vertritt Freud die Ansicht, dass bei der Errichtung des Über-Ichs die Agressionstriebe, die im «Todestrieb» wurzeln, mitwirkten. Wir müssen uns somit auch mit diesem näher befassen.

In seiner früheren Arbeit «Jenseits des Lustprinzips» kommt Freud zu dem Schluss, dass im Grunde genommen zwischen dem Selbsterhaltungstrieb und dem Sexualtrieb kein Gegensatz besteht, da beide «die Teile der lebenden Substanz zueinander zu drängen und zusammenzuhalten suchen». ${ }^{2}$ So entstand die übergeordnete Bezeichnung «Eros» für den allgemeinen Begriff Lebenstrieb. Dem Eros untergeordnete Begriffe waren der Ich- und die eigentlichen Sexualtriebe (Objekttriebe), beide libidinöser Natur.

Freud meint, dass vieles darauf hindeutet, dass es auch im Seelenleben etwas wie ein Trägheitsgesetz gibt, einen Wiederholungszwang, der sich über das Lustprinzip hinwegsetzt. So weist er auf die Erblichkeit hin und auf die Tatsache, dass der Keim eines Tieres «genötigt ist», in seiner Entwicklung die Strukturen all der Formen, von denen das Tier abstammt, zu durchlaufen. Auch das Regenerationsvermögen, das ein verlorenes Organ durch ein neues ersetzt, gehört hierher. Ebenso könnte man die «Macht der Gewohnheit» in diesem Zusammenhang erwähnen.

Da es der konservativen Natur der Triebe widersprechen würde, wenn das Ziel des Lebens ein nie vorher erreichter Zustand wäre, kommt Freud zu der Schlussfolgerung, dass das Ziel alles Lebenden nur der Tod sein kann.

Freud verweist auch auf die Arbeiten des bekannten Biologen Weissmann, der in der lebenden Substanz zwischen dem sterblichen Körper im engeren Sinn, dem «Soma», und der unsterblichen Keimzelle unterscheidet. Auch erwähnt er die Theorie Herings von den aufbauenden, assimilatorischen, und den abbauenden, dissimilatorischen Prozessen, die in der lebenden Substanz in entgegengesetzter Richtung verlaufen. War somit die Lehre Freuds ursprünglich eine monistische, wandelte sie sich zu einer dualistischen: dem «Eros», der das lebenerhaltende Prinzip verkörpert, wurde der Sadismus, wurden die Agressionstriebe, welche dem Todestrieb entstammen, gegenübergestellt. Hiedurch erhält die Lehre Freuds eine pessimistische Färbung, sagt doch Freud selbst im «Jenseits des Lustprinzips», dass er «unversehens 
in den Hafen der Philosophie Schopenhauers» ${ }^{3}$ eingelaufen ist, die man bestimmt keine optimistische nennen kann.

Wie bereits erwähnt, beschäftigt sich Freud in seiner Abhandlung «Das Unbehagen in der Kultur» mit dem Anteil, den die Agressionstriebe bei der Errichtung des Über-Ichs hatten.

Bei diesem Vorgang wird nach Freud die Agression verinnerlicht, «introjiziert» und von einem Teil des Ichs übernommen, das sich als Über-Ich dem übrigen entgegenstellt und als «Gewissen» gegen das Ich dieselbe Agressionsbereitschaft ausübt, die das Ich gerne an anderen Individuen befriedigt hätte. Aus der Spannung zwischen dem Über-Ich und dem Ich resultiert das Schuldbewusstsein, das sich als Strafbedürfnis äussert. Ursprünglich wird auf eine Triebbefriedigung aus Angst vor dem Liebesverlust verzichtet. Verliert man die Liebe, büsst man auch den Schutz des Übermächtigen ein, von dem man abhängig ist. Eine wichtige Änderung tritt ein, wenn die äussere Autorität durch die Aufrichtung des Über-Ichs verinnerlicht wird. Jetzt entfällt der Unterschied zwischen Böses tun und Böses wollen, denn vor dem Über-Ich kann man nichts verbergen, auch nicht Gedanken. Die äussere Autorität zwingt zum Verzicht auf Triebbefriedigung, die innere, das Über-Ich drängt, da man vor dem Über-Ich nichts verbergen kann, zur Bestrafung. Im zweiten Falle, im Gegensatz zum ersteren, hilft auch der Triebverzicht nicht, denn der Wunsch bleibt bestehen und lässt sich vor dem Über-Ich nicht verheimlichen. Der Triebverzicht hat hier somit keine befreiende Wirkung, noch mehr, die Enthaltsamkeit steigert den Wunsch nach Triebbefriedigung, damit aber auch das Schuldbewusstsein. Dies die Erklärung, warum gerade jene, die es in der Heiligkeit am weitesten gebracht haben, sich der grössten Sündhaftigkeit bezichtigen.

Die Strenge der äusseren Autorität wird somit von dieser teilweise abgelöst und auf die neu errichtete innere, das Über-Ich, übertragen. Die Agression des Gewissens ist somit die fortgesetzte Strenge der äusseren Autorität. Aber Freud glaubt, dass es noch einen anderen Ursprung der Agression des Über-Ichs gibt. Gegen die Autorität, die das Kind an einer Befriedigung hindert, muss sich bei diesem ein erhebliches Mass von Agressionsneigung gegen die Autorität, die das Verbot ausspricht, entwickeln. Notgedrungen muss das Kind auf die Befriedigung dieser rachesüchtigen Agression verzichten. Es hilft sich auf die Weise, dass es die äussere unangreifbare Autorität durch Identifizierung in sich aufnimmt, die nun das Über-Ich wird und in den Besitz all der Agression gerät, die das Kind gegen sie ausgeübt hätte. Es ist so, als ob das Kind sagte: «Wenn ich der Vater wäre und Du das Kind, ich würde dich schlecht behandeln.» «Das Ich des Kindes», sagt Freud, «muss sich auf diese Weise mit der Rolle des erniedrigten Vaters begnügen». ${ }^{4}$ 
So befriedigt das Kind durch Umkehrung der realen Situation seine Rache- und Agressionsgelüste gegen den Vater, der es zum Triebverzicht zwingt. Jeder neu erzwungene Verzicht auf Triebbefriedigung verstärkt die Agression des Über-Ichs.

Den eigentlichen Ursprung aller dieser Vorgänge sieht Freud in der ambivalenten Einstellung des Kindes zum Vater. So haben wir letzten Endes auch hier mit dem Gegensatz von Liebe und Hass, von Eros und Todestrieb zu tun.

Aber ob positiv oder negativ, ob Hass oder Liebe, ob Lebens- oder Todestrieb, Triebe bleiben Triebe. Da wir aber zur Einsicht gekommen sind, dass das Triebhafte in uns den «freien» Willen ausschliesst, kann auch der Umstand, dass an unseren Entscheidungen und Handlungen das Über-Ich einen Anteil hat, uns zur Annahme eines «freien» Willens keineswegs veranlassen.

\section{Das Realitätsprinzip und der freie Wille}

Wir haben gesehen, dass ganz gleichgültig, ob das Es oder aber das Über-Ich an unseren Entscheidungen und Handlungen den grösseren Anteil hat, man von der Betätigung eines freien Willens in keiner Weise sprechen kann.

An unseren Willensäusserungen und Handlungen ist aber auch unser Ich beteiligt, welches das Realitätsprinzip vertritt. Es frägt sich, ob nicht schon dieser letztere Umstand allein uns zur Annahme eines freien Willens berechtigt?

Unsere Triebregungen werden in unserem Ich wahrgenommen, werden uns bewusst. Jedes Lebewesen ist in hohem Masse von seiner Umwelt abhängig. Wir erhalten die Nahrung von aussen und wir können uns gegen Kälte nur auf die Weise schützen, dass wir uns mit Kleidern versehen und uns Wohnbauten errichten. Auch die Libido verschafft sich auf die Art eine Befriedigung, dass sie sich der Objekte bemächtigt, sie besetzt. So liegen auch unsere Triebziele, insoweit es sich nicht um eine rein narzistische Befriedigung handelt, ausserhalb uns und sind somit diese durch die Verhätlnisse, welche wir in der realen Welt vorfinden, bedingt.

Das Ich ist bestrebt unter Anwendung des Realitätsprinzips auf ökonomischste, d.h. einfachste und leichteste Weise, die angestrebten Ziele zu erreichen.

Das Realitätsprinzip fordert von uns die Anpassung an die Aussenwelt, d.h. die Unterordnung unter Gesetze, die in der realen Welt herrschen. Den logischen Schlussfolgerungen, denen wir folgen müssen, um ein Triebziel zu erreichen, haftet ein Zwang an, dem wir nicht entrinnen können, ohne das 
Realitätsprinzip als solches fallen zu lassen, damit aber auch auf das Triebziel zu verzichten. Vielleicht empfinden wir nichts schmerzhafter und fühlen uns niemals «unfreier», als wenn wir, den in der realen Welt obwaltenden Umständen Rechnung tragend, auf die Befriedigung unserer Begierden verzichten.

In jenen Fällen aber, wo wir uns gegen die Vernunft versündigen, geschieht dies nicht, weil unser Wille frei ist, sondern weil wir entweder die gegebene Situation nicht klar genug übersehen, wir Denkfehler begehen oder aber gegen unsere eigene bessere Einsicht, unseren Trieben dermassen verfallen sind, dass sie das Realitätsprinzip durchbrechen und es somit nicht zur Geltung kommen lassen. Im ersteren Falle sind es die Unzulänglichkeiten unseres Denkvermögens und unser Unvermögen, die reale Welt in ihrem vollen Umfange und in allen ihren Einzelheiten zu erfassen, im zweiten die Übermacht unserer Triebe, welche unsere Handlungsweise bestimmen, determinieren.

Der Zwang aber, der dem Realitätsprinzip anhaftet, kann auch so stark und so unerträglich werden, dass das Realitätsprinzip und mit diesem die Realität selbst fallen gelassen wird und dann Menschen in den Wahnsinn flüchten.

So gibt uns bei näherer Betrachtung auch das Realitätsprinzip keinerlei Handhabe zur Annahme eines irgendwo ausserhalb der Kausalität schwebenden «freien» Willens.

Freud meint, dass kulturelle Errungenschaften durch Triebverzicht bedingt sind.

Nun haben wir in den letzten Jahrhunderten auf naturwissenschaftlichem und auf technischem Gebiet so viele Erfahrungen gesammelt und so viele Kenntnisse angehäuft, dass der Fortschritt auf diesen Gebieten, im Vergleich zu den Anfängen der menschlichen Kultur, uns verhältnismässig sehr leicht gemacht wird. Er vollzieht sich nach den dem Realitätsprinzip selbst innewohnenden Gesetzen, ohne dass es im Sinne Freuds, eines Triebverzichtes bedarf. Vielleicht ist dies die Erklärung, warum über die Diskrepanz zwischen Kultur und Zivilisation geklagt wird.

Das Realitätsprinzip zeigt uns nicht nur, wie wir die Naturkräfte bezwingen können, sondern gibt uns auch die Möglichkeit, gegen unsere Mitmenschen Gewaltmittel anzuwenden, die mit den Fortschritten der naturwissenschaftlichen und technischen Forschung immer gewaltiger werden.

Das Realitätsprinzip sollte ursprünglich dem Eros, im weiteren Sinne Freuds, also dem Selbsterhaltungs- und Sexualtrieb dienen, konnte aber auch den Agressionstrieben nutzbar gemacht werden und half fremdes Leben zu vernichten. Obwohl Freud an das Vorhandensein eines Vervollkommnungs- 
triebes nicht glaubt, gibt er doch zu, dass die bisherige Entwicklung der Menschheit unverkennbar eine Tendenz nach oben aufweist. Sollte aber die Gefahr eines Atomkrieges und die Vernichtung der Menschheit durch diesen Wirklichkeit werden, stünde man wahrlich vor einem unlösbaren Rätsel, und man müsste sich fragen, wozu dann das Realitätsprinzip ins Leben gerufen wurde. Es bleibt uns mithin nichts anderes übrig, als zu hoffen, dass die aufbauenden Kräfte auch weiterhin stärker sein werden als die destruktiven, denn wir wollen nicht annehmen, dass das Realitätsprinzip, dessen Aufgabe es ursprünglich war, das Leben zu schützen und zu fördern, letzten Endes dazu bestimmt sein sollte, dieses Leben zu zerstören und zu vernichten.

\section{Die «Weltanschauung» als subjektive Wertordnung}

In seiner schon früher erwähnten Arbeit «Das Unbehagen in der Kultur» spricht Freud von dem Bestreben der Menschen, Unlustgefühle zu vermeiden und Lustgefühle - das «Glück» im engeren Sinne - zu erleben. Freud zweifelt an der Zweckdienlichkeit dieses Programms, da es im Hader mit der ganzen Welt liegt, mit dem Makrokosmos ebenso wie mit dem Mikrokosmos. Dieses Programm ist nach Freud überhaupt nicht ausführbar und widerspricht allen Einrichtungen des Alls. «Die Absicht, dass der Mensch glücklich sei», sagt Freud, «ist im Plan der Schöpfung nicht enthalten». ${ }^{5}$ Wir sind so eingerichtet, dass wir nur den Kontrast intensiv geniessen können, einen befriedigenden Zustand nur wenig. Freud verweist auf Goethe, der mahnt: «Nichts ist schwerer zu ertragen, als eine Reihe von schönen Tagen ${ }^{6}$, obschon Freud darin eine Übertreibung erblickt. Um wievieles leichter hat es hingegen mit uns das Unglück. Von drei Seiten drohen die Leiden: vom eigenen Körper her, von der Aussenwelt und aus den Beziehungen zu anderen Menschen. Die Leiden aus letzterer Quelle empfinden wir vielleicht am schmerzlichsten.

Hier haben wir es tatsächlich schwierig. Wenn unsere Interessen mit den fremden kollidieren, halten wir es für recht und billig, dass das fremde Lustprinzip unserem eigenen weicht.

Es ist auch bemerkenswert, dass das Lustprinzip eigentlich nur die Gegenwart und die Zukunft, nicht aber die Vergangenheit kennt. Vergangene Freude und vergangenes Leid empfinden wir als etwas Traumhaftes, fast Irreales. Dies gilt für uns selbst und natürlich in noch höherem Masse bei Beurteilung der Freuden und Leiden unserer Mitmenschen. Aber die Gegenwart verwandelt sich jeden Augenblick in die Vergangenheit und alles, was vor uns liegt, wird einmal hinter uns liegen. Wie soll man aber dann, wenn 
man dies alles berücksichtigt, eine objektive Rangordnung der Werte aufstellen? Wir wollen auch von einer solchen absehen und uns der subjektiven Wertordnung, die ihren Ausdruck in der «Weltanschauung» findet, zuwenden.

Die Widersprüche und die Unzulänglichkeiten der menschlichen Natur, die Unzulänglichkeit des Lustprinzips als solchen, erzeugen in uns ein latentes Gefühl des Unbehagens. «La prévoyance, la prévoyance, voilà la source de toutes nos souffrances» (Die Voraussicht, die Voraussicht, das ist die Quelle all unserer Leiden $)^{7}$ meinte schon Jean Jacques Rousseau. Der Zug nach dem Absoluten im Menschen ist unverkennbar. Je mehr das Realitätsprinzip um sich greift und im menschlichen Leben eine immer grössere Rolle spielt, desto besser verstehen wir es die Naturkräfte zu beherrschen und uns nutzbar zu machen, desto mehr verlieren wir aber die innere Verbundenheit mit ihnen. Die Sehnsucht nach dem Primitiven und Metaphysischen in der modernen bildenden Kunst entspringt vielleicht gerade dem Wunsche, dieser Vereinsamung im Weltall zu entrinnen.

So stellt die Weltanschauung einen Versuch dar, die auseinanderstrebenden Kräfte im Menschen unter der Führung des Realitätsprinzips zu einer Einheit zusammenzufassen. Aber es ist nicht leicht, das Unversöhnliche in uns und ausserhalb uns zu versöhnen. Jede Weltanschauung ist daher eine Kompromisslösung, zum Teil auf Kosten des Realitätsprinzips selbst, das bei jeder Weltanschauung wegweisend und massgebend sein sollte. Da aber die Weltanschauung uns von allerlei Zweifel befreien und unsere Entschlusskraft und Aktionsfähigkeit stärken soll, dass das Bild, das wir uns von der Aussenwelt machen, möglichst vereinfacht und mit unserer inneren Welt, mit allen ihren Wünschen, Hoffnungen und allen sonstigen uns innewohnenden Eigenheiten in Einklang gebracht werden. Nicht umsonst hat man den von

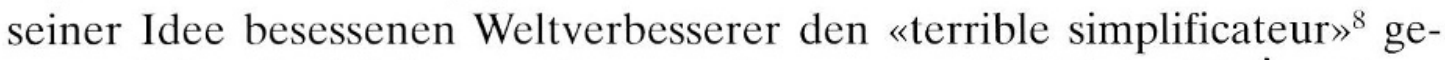
nannt. Diese Projektion von innen nach aussen verleiht jeder Weltanschauung, aber auch jeder Philosophie ein individuelles und subjektives Gepräge. Es ist selbstverständlich, dass hiebei für Rationalisierungen ein breiter Spielraum offenbleibt. Sie bestehen darin, dass Motive, die unserem Triebleben entstammen, dem Über-Ich zugeschoben oder, als dem Realitätsprinzip entsprechend und von diesem gefordert, umgedeutet werden. Wie bequem z.B. ist der Satz, wonach der Zweck die Mittel heilige. Die bösen Mittel setzen ohne Aufschub ein, die guten Ziele hingegen liegen in weiter Ferne und sind nebulös. Meistens ist auch eine Vergleichsmöglichkeit der beiden Grössen nicht gegeben, da es an einem geeigneten tertium comparationis fehlt. Eine Weltanschauung kann auch einen Grad von Starrheit erreichen, dass sie paranoidalen Charakter annimmt. Dies ist beim religiösen oder beim politi- 
schen Fanatiker, bei einem überspitzten Nationalgefühl, beim Antisemitismus und dgl. der Fall. Da es ferner natürlich ist, dass Menschen mit gleicher Weltanschauung und Gesinnung sich zu grösseren Gemeinschaften zusammenschliessen, kommt dann zu dem vorhin gesagten auch noch alles hinzu, was zum Kapitel «Massenpsychologie» gehört.

Unsere Weltanschauung bestimmt zweifellos auch unsere Handlungsweise. Da sie aber, im Sinne der Dreiteilung Freuds, ein Ergebnis der Betätigung der bereits besprochenen drei Instanzen, nämlich des Es, des Ichs und des Über-Ichs darstellt, lässt auch sie keinen Spielraum für einen ausserhalb der Kausalitätsreihe liegenden «freien» Willen zu.

1 Franz Alexander und Hugo Staub: Der Verbrecher und seine Richter, Wien, Internationaler Psychoanalytischer Verlag, 1929, 45.

2 Sigmund Freud: Jenseits des Lustprinzips, Gesammelte Werke, Bd. XIII, S. 66, Anm. 1. Sergius Pankejeff zitiert hier falsch. Der Originaltext von Freud lautet «[Mit der Aufstellung der narzißtischen Libido und der Ausdehnung des Libidobegriffes auf die einzelne Zelle wandelte sich uns der Sexualtrieb zum Eros, der] die Teile der lebenden Substanz zueinanderzudrängen und zusammenzuhalten sucht.»

3 Ibid., S. 53.

4 Sigmund Freud: Das Unbehagen in der Kultur, Gesammelte Werke, Bd. XIV, S. 489. Sergius Pankejeff zitiert falsch. Der Originaltext lautet: «Das Ich des Kindes muß sich mit der traurigen Rolle der so erniedrigten Autorität - des Vaters - begnügen.»

5 Ibid., S. 434. Sergius Pankejeff lässt hier die von Freud gesetzten Klammern aus: «Die Absicht, dass der Mensch 'glücklich' sei, ist im Plan der 'Schöpfung' nicht enthalten.»

6 Von Freud zitiert, ibid.

7 Sergius Pankejeff zitiert diesen Satz auch in seinen Memoiren, Der Wolfsmann vom Wolfsmann, herausgegeben von Muriel Gardiner, Fischer Verlag, Frankfurt am Main, 1972, 176.

8 «Schlimmen Vereinfacher.» 